\title{
Existence Results for Constrained Quasivariational Inequalities
}

\author{
V. V. Motreanu \\ Department of Mathematics, Ben Gurion University of the Negev, Be’er Sheva 84105, Israel \\ Correspondence should be addressed to V. V. Motreanu; motreanu@post.bgu.ac.il
}

Received 18 June 2013; Accepted 4 September 2013

Academic Editor: Rodrigo Lopez Pouso

Copyright (c) 2013 V. V. Motreanu. This is an open access article distributed under the Creative Commons Attribution License, which permits unrestricted use, distribution, and reproduction in any medium, provided the original work is properly cited.

We deal with a constrained quasivariational inequality under a general form. We study existence of solutions in two situations depending on whether the set of constraints is bounded or possibly unbounded.

\section{Introduction and Statement of Main Results}

Let $X$ be a real reflexive and separable Banach space assumed to be compactly embedded in a Banach space $Y$. We denote by $X^{*}$ the dual space of $X$, by $Y^{*}$ the dual space of $Y$, by $\langle\cdot, \cdot\rangle_{X}$ the duality brackets between $X^{*}$ and $X$, by $\langle\cdot, \cdot\rangle_{Y}$ the duality brackets between $Y^{*}$ and $Y$, by $\|\cdot\|_{X}$ the norm of $X$, and by $\|\cdot\|_{Y}$ the norm of $Y$. Given a function $\psi: X \rightarrow \mathbb{R} \cup\{+\infty\}$, we denote by $D(\psi):=\{x \in X: \psi(x)<+\infty\}$ the effective domain of $\psi$.

In this paper we deal with the following problem

Find $u \in K$ such that $(u, u) \in D(\Phi)$,

$$
\begin{gathered}
\langle A u, v-u\rangle_{X}+\Phi(u, v)-\Phi(u, u)+J^{0}(u ; v-u) \\
\geq\langle f, v-u\rangle_{X}, \quad \forall v \in K .
\end{gathered}
$$

We describe the data entering problem (1):

(i) $K \subset X$ is a nonempty, convex, closed subset;

(ii) $A: X \rightarrow X^{*}$ is a (possibly nonlinear) operator;

(iii) $\Phi: X \times X \rightarrow \mathbb{R} \cup\{+\infty\}$ is such that, for all $\eta \in$ $K$, the function $\Phi(\eta, \cdot): X \rightarrow \mathbb{R} \cup\{+\infty\}$ is convex with $K \cap D(\Phi(\eta, \cdot)) \neq \emptyset$; moreover, we will denote by $\partial \Phi(\eta, \cdot)$ the convex subdifferential of $\Phi(\eta, \cdot)$; that is,

$$
\begin{aligned}
\partial \Phi(\eta, u)=\left\{w \in X^{*}:\right. & \Phi(\eta, v)-\Phi(\eta, u) \\
& \left.\geq\langle w, v-u\rangle_{X}, \forall v \in X\right\} ;
\end{aligned}
$$

(iv) $J: Y \rightarrow \mathbb{R}$ is a locally Lipschitz function, and the notation $J^{0}$ stands for its generalized directional derivative in the sense of Clarke [1]; that is,

$$
\begin{aligned}
J^{0}(u ; v) & =\limsup _{\substack{w \rightarrow u \\
\lambda \rightarrow 0^{+}}} \frac{J(w+\lambda v)-J(w)}{\lambda}, \quad \forall u, v \in Y .
\end{aligned}
$$

In addition, we will denote by $\partial J$ the generalized gradient of $J$; that is,

$\partial J(u)$

$$
=\left\{w \in Y^{*}: J^{0}(u ; v) \geq\langle w, v\rangle_{Y}, \forall v \in Y\right\}, \quad \forall u \in Y ;
$$

(v) $f \in X^{*}$.

Problem (1) is called a constrained quasivariational problem. Typically, we can choose $X$ to be the Sobolev space $\left(H_{0}^{1}(\Omega),\|\nabla \cdot\|_{L^{2}(\Omega)}\right)$ defined as the closure of $C_{c}^{\infty}(\Omega)$ in $H^{1}(\Omega)$ for a bounded domain $\Omega \subset \mathbb{R}^{N}(N \geq 1), Y$ to be the Lebesgue space $L^{p}(\Omega)$ for $1 \leq p<2^{*}$ (where $2^{*}=$ $+\infty$ if $N \in\{1,2\}$ and $2^{*}=2 N /(N-2)$ if $\left.N \geq 3\right)$, $K=\left\{u \in H_{0}^{1}(\Omega): u \geq 0\right.$ a.e. in $\left.\Omega\right\}, A=-\Delta$ (the negative Laplacian operator), $\Phi(u, v)=\int_{\Omega} g(u, v) d x$ where $g: \mathbb{R}^{2} \rightarrow \mathbb{R}_{+}$is convex in the second variable (then $\left.D(\Phi)=\left\{(u, v) \in H_{0}^{1}(\Omega) \times H_{0}^{1}(\Omega): g(u, v) \in L^{1}(\Omega)\right\}\right)$, and 
$J(u)=\int_{\Omega} j(x, u(x)) d x$ where $j: \Omega \times \mathbb{R} \rightarrow \mathbb{R}$ is locally Lipschitz in the second variable. Constrained quasivariational problems were extensively studied; we refer, for example, to $[2-5]$ and to the references therein. We point out three aspects which make our approach natural and general. First, we deal with the general setting of a pair of Banach spaces $(X, Y)$ instead of focusing on spaces of functions; in particular, our results can be applied to problems with different boundary conditions. Second, the set of constraints $K$ may be unbounded. Third, the form of the studied problem allows both variational and hemivariational constraints as it involves both a convex term $\Phi(u, \cdot)$ and a generalized directional derivative $J^{0}$; this type of problems models important processes in mechanics and engineering (see $[6,7]$ ).

In this paper, we consider the following hypotheses on the data described above:

$\left(H_{1}\right)$ for every sequence $\left\{u_{n}\right\}_{n \geq 1} \subset K$ with $u_{n} \rightarrow u$ in $X$, for some $u \in K$, one has

$$
\begin{aligned}
& \langle A u, u-v\rangle_{X} \\
& \quad \leq \limsup _{n \rightarrow \infty}\left\langle A u_{n}, u_{n}-v\right\rangle_{X}, \quad \forall v \in K
\end{aligned}
$$

$\left(H_{2}\right)$ whenever $\left\{\left(\eta_{n}, u_{n}\right)\right\}_{n \geq 1} \subset(K \times K) \cap D(\Phi), \eta_{n} \rightarrow \eta$ in $X, u_{n} \rightarrow u$ in $X$, one has $(\eta, u) \in(K \times K) \cap D(\Phi)$ and

$$
\begin{aligned}
\limsup _{n \rightarrow \infty}\left(\Phi\left(\eta_{n}, v\right)-\Phi\left(\eta_{n}, u_{n}\right)\right) \\
\leq \Phi(\eta, v)-\Phi(\eta, u), \quad \forall v \in K ;
\end{aligned}
$$

$\left(H_{3}\right)$ given $\eta \in K$, if $u_{1}, u_{2} \in K$ satisfy $\left(\eta, u_{1}\right) \in D(\Phi)$, $\left(\eta, u_{2}\right) \in D(\Phi)$ and

$$
\begin{gathered}
J^{0}\left(\eta ; u_{2}-u_{1}\right)+J^{0}\left(\eta ; u_{1}-u_{2}\right) \\
\geq\left\langle A u_{2}-A u_{1}, u_{2}-u_{1}\right\rangle_{X},
\end{gathered}
$$

$$
\text { then } u_{1}=u_{2} \text {. }
$$

Remark 1. We emphasize certain situations when hypotheses $\left(H_{1}\right)-\left(H_{3}\right)$ are satisfied.

(a) Hypothesis $\left(H_{1}\right)$ is satisfied, for instance, if $A$ is weakly strongly continuous, that is, $A$ is continuous from $X$ endowed with the weak topology to $X^{*}$ endowed with the norm topology.

(b) Note that $\left(H_{1}\right)$ is satisfied, for instance, for $X=$ $H_{0}^{1}(\Omega)$, any closed, convex subset $K \subset X$, and $A: H_{0}^{1}(\Omega) \rightarrow$ $H_{0}^{1}(\Omega)^{*}$ defined by $A=-\Delta$, where $\Delta: H_{0}^{1}(\Omega) \rightarrow H_{0}^{1}(\Omega)^{*}$ is the Laplacian operator, with $\Omega \subset \mathbb{R}^{N}(N \geq 1)$ a bounded domain. Indeed, let a sequence $\left\{u_{n}\right\}_{n \geq 1} \subset K$ with $u_{n} \rightarrow u$ in
$H_{0}^{1}(\Omega)$, for some $u \in K$. Using the weak lower semicontinuity of the norm, we can write

$$
\begin{aligned}
\limsup _{n \rightarrow \infty}\left\langle-\Delta u_{n}, u_{n}-v\right\rangle & =\limsup _{n \rightarrow \infty}\left(\left\|u_{n}\right\|_{H_{0}^{1}(\Omega)}^{2}-\left(u_{n}, v\right)_{H_{0}^{1}(\Omega)}\right) \\
& \geq \liminf _{n \rightarrow \infty}\left\|u_{n}\right\|_{H_{0}^{1}(\Omega)}^{2}-(u, v)_{H_{0}^{1}(\Omega)} \\
& \geq\|u\|_{H_{0}^{1}(\Omega)}^{2}-(u, v)_{H_{0}^{1}(\Omega)} \\
& =\langle-\Delta u, u-v\rangle
\end{aligned}
$$

for all $v \in H_{0}^{1}(\Omega)$. Here, $\langle\cdot, \cdot\rangle$ are the duality brackets for the pair $\left(H_{0}^{1}(\Omega)^{*}, H_{0}^{1}(\Omega)\right)$ and $(u, v)_{H_{0}^{1}(\Omega)}=\int_{\Omega} \nabla u \cdot \nabla v d x$ denotes the scalar product on $H_{0}^{1}(\Omega)$. Whence $\left(H_{1}\right)$ holds in this case.

(c) Hypothesis $\left(\mathrm{H}_{2}\right)$ is fulfilled in the case where $\Phi$ is sequentially weakly lower semicontinuous, $D(\Phi)$ is weakly closed, and $\Phi(\cdot, u)$ is weakly strongly continuous on its effective domain for all $u \in X$.

(d) If $A$ is strongly monotone, that is, there exists a constant $m>0$ such that

$$
\left\langle A u_{2}-A u_{1}, u_{2}-u_{1}\right\rangle_{X} \geq m\left\|u_{1}-u_{2}\right\|_{X}^{2}, \quad \forall u_{1}, u_{2} \in K,
$$

and $\partial J$ is bounded on $K$ in the sense that

$$
\|\zeta\|_{Y^{*}} \leq c\|u\|_{Y}, \quad \forall \zeta \in \partial J(u), \quad \forall u \in K,
$$

with a positive constant $c<m /(2 \bar{c})$, where $\bar{c}>0$ is the best constant satisfying $\|u\|_{Y} \leq \bar{c}\|u\|_{X}$, for all $u \in X$ (which exists by the continuity of the embedding of $X$ in $Y$ ), then condition $\left(\mathrm{H}_{3}\right)$ is satisfied.

(e) If $A$ is strictly monotone and $J$ is Gâteaux differentiable and regular (see [1, Definition 2.3.4]), then condition $\left(\mathrm{H}_{3}\right)$ is satisfied. In particular, if $\mathrm{A}$ is strictly monotone and $J$ is continuously differentiable, then $\left(\mathrm{H}_{3}\right)$ is satisfied.

In this paper, we distinguish two cases depending on whether the set $K$ is bounded or not necessarily bounded. The following result concerns the former situation.

Theorem 2. Assume that conditions $\left(H_{1}\right)-\left(H_{3}\right)$ are satisfied and that the closed, convex set $K$ is bounded in $X$. Then problem (1) has at least one solution.

Remark 3. Note that the existence of a solution of problem (1), which is the conclusion of Theorem 2 , forces the intersection $\operatorname{diag}(K) \cap D(\Phi)$ to be nonempty, where the notation $\operatorname{diag}(K)$ stands for the diagonal of the set $K$; that is, $\operatorname{diag}(K)=$ $\{(v, v): v \in K\}$. The nonemptiness of this intersection is not directly implied by the hypotheses $\left(H_{1}\right)-\left(H_{3}\right)$, nor by the assumption made that $K \cap D(\Phi(\eta, \cdot)) \neq \emptyset$ for all $\eta \in$ $K$. However, Theorem 4 below incorporates hypothesis $\left(H_{4}\right)$ which assumes in particular that $\operatorname{diag}(K) \cap D(\Phi) \neq \emptyset$. 
Now, we deal with the case where $K$ is not assumed to be bounded. In this case, we additionally suppose the following:

$\left(H_{4}\right)$ there exist an element $v_{0} \in K$ with $\left(\eta, v_{0}\right) \in D(\Phi)$ for all $\eta \in K$ and a real $p \geq 1$ such that

$$
\limsup _{\|w\|_{X} \rightarrow \infty} \frac{\left\langle A w, w-v_{0}\right\rangle_{X}}{\|w\|_{X}^{p}}=+\infty ;
$$

$\left(H_{5}\right)$ there exists a constant $c_{0}>0$ such that we have

$$
\begin{aligned}
& \left\langle z, v_{0}-u\right\rangle_{X} \\
& \quad \leq c_{0}\left(1+\|u\|_{X}^{p}\right), \quad \forall z \in \partial \Phi(u, \cdot)\left(v_{0}\right), \\
& \|z\|_{Y^{*}} \leq c_{0}\left(1+\|u\|_{Y}^{p-1}\right), \quad \forall z \in \partial J(u),
\end{aligned}
$$

for all $u \in K$ with $(u, u) \in D(\Phi)$, where $v_{0}$ and $p \geq 1$ are as in $\left(H_{4}\right)$.

We state now our main result for problem (1) dealing with the case where the set $K$ is possibly unbounded.

Theorem 4. Assume that conditions $\left(H_{1}\right)-\left(H_{5}\right)$ are satisfied. Then problem (1) has at least a solution.

The rest of the paper is organized as follows. In Section 2, we present the proof of Theorem 2, where we apply a version of the Schauder fixed point theorem. In Section 3, we give the proof of Theorem 4, which is actually based on Theorem 2 .

\section{Proof of Theorem 2}

For each $\eta \in K$, we consider the auxiliary problem

Find $u \in K$ such that $(\eta, u) \in D(\Phi)$,

$$
\begin{gathered}
\langle A u, v-u\rangle_{X}+\Phi(\eta, v)-\Phi(\eta, u)+J^{0}(\eta ; v-u) \\
\geq\langle f, v-u\rangle_{X}, \quad \forall v \in K .
\end{gathered}
$$

Our first purpose, accomplished in Lemma 6 below, is to show that problem (13) has a unique solution. To do this, we need Fan's lemma (see [8, page 208]) which we recall in the following statement.

Theorem 5. Let $W$ be a Hausdorff topological vector space, let $Z$ be a nonempty subset of $W$, and let $F: Z \rightarrow 2^{W}$ be such that

(i) $F(x)$ is a nonempty, closed subset of $W$, for all $x \in Z$;

(ii) conv $\left\{x_{1}, \ldots, x_{n}\right\} \subset \bigcup_{i=1}^{n} F\left(x_{i}\right)$ for all $\left\{x_{1}, \ldots, x_{n}\right\} \subset$ $Z$;

(iii) there is $\bar{x} \in Z$ for which $F(\bar{x})$ is compact.

Then $\bigcap_{x \in Z} F(x) \neq \emptyset$.

Lemma 6. Assume that hypotheses $\left(\mathrm{H}_{1}\right)-\left(\mathrm{H}_{3}\right)$ are fulfilled and that the closed, convex set $K$ is bounded in $X$. Then, for every $\eta \in K$, problem (13) has a unique solution.
Proof. Fix $\eta \in K$. Consider the set-valued mapping $G: K \cap$ $D(\Phi(\eta, \cdot)) \rightarrow 2^{X}$ defined by

$$
\begin{aligned}
G(v)=\{u \in K \cap D(\Phi(\eta, \cdot)): & \langle A u-f, u-v\rangle_{X} \\
& -J^{0}(\eta ; v-u) \\
& +\Phi(\eta, u)-\Phi(\eta, v) \leq 0\}
\end{aligned}
$$

for all $v \in K \cap D(\Phi(\eta, \cdot))$. We show that the assumptions of Theorem 5 are satisfied for $W=X$ endowed with the weak topology, $Z=K \cap D(\Phi(\eta, \cdot))$, and $F=G$.

For every $v \in K \cap D(\Phi(\eta, \cdot))$, we clearly have $v \in G(v)$; hence $G(v)$ is nonempty.

We check that $G(v)$ is weakly compact for every $v \in K \cap$ $D(\Phi(\eta, \cdot))$. To this end, we first prove that $G(v)$ is sequentially weakly closed in $X$. Let a sequence $\left\{u_{n}\right\}_{n \geq 1} \subset G(v)$ with $u_{n} \rightarrow u$ in $X$, for some $u \in X$. Taking into account that $X$ is compactly embedded in $Y$ it follows that $u_{n} \rightarrow u$ in $Y$. Using the first part of assumption $\left(\mathrm{H}_{2}\right)$, we have that $u \in K \cap D(\Phi(\eta, \cdot))$. As $u_{n} \in G(v)$, we know that

$$
\begin{aligned}
& \left\langle A u_{n}, u_{n}-v\right\rangle_{X} \\
& \quad \leq\left\langle f, u_{n}-v\right\rangle_{X}+J^{0}\left(\eta ; v-u_{n}\right)+\Phi(\eta, v)-\Phi\left(\eta, u_{n}\right) .
\end{aligned}
$$

Passing to the lim sup as $n \rightarrow \infty$, we find

$$
\begin{aligned}
& \limsup _{n \rightarrow \infty}\left\langle A u_{n}, u_{n}-v\right\rangle_{X} \\
& \quad \leq\langle f, u-v\rangle_{X}+J^{0}(\eta ; v-u)+\Phi(\eta, v)-\Phi(\eta, u) .
\end{aligned}
$$

Here we made use of the weak convergence $u_{n} \rightarrow u$ in $X$, the continuity of $J^{0}(\eta ; \cdot)$ on $Y$, and the second part of $\left(H_{2}\right)$. Combining with $\left(H_{1}\right)$, we obtain that $u \in G(v)$, thereby $G(v)$ is sequentially weakly closed in $X$.

Using that $X$ is reflexive and separable and $K$ is bounded, convex, and closed, we deduce that $K$ is metrizable and weakly compact (see, e.g., [9, pages 44-50]). Since $G(v) \subset K$ and using that $G(v)$ is sequentially weakly closed, we derive that $G(v)$ is weakly compact whenever $v \in K \cap D(\Phi(\eta, \cdot))$. Therefore conditions (i) and (iii) in Theorem 5 are fulfilled.

We focus now on the verification of condition (ii) in Theorem 5. Arguing by contradiction, we suppose that there exist $v_{1}, \ldots, v_{n} \in K \cap D(\Phi(\eta, \cdot))$ and $u_{0} \in \operatorname{conv}\left\{v_{1}, \ldots, v_{n}\right\}$ such that $u_{0} \notin \bigcup_{i=1}^{n} G\left(v_{i}\right)$. The convexity of the set $K$ and of the function $\Phi(\eta, \cdot)$ ensures that $u_{0} \in K \cap D(\Phi(\eta, \cdot))$. Then the assertion that $u_{0} \notin \bigcup_{i=1}^{n} G\left(v_{i}\right)$ reads as

$$
\begin{aligned}
\left\langle A u_{0}\right. & \left.-f, u_{0}-v_{i}\right\rangle_{X}-J^{0}\left(\eta ; v_{i}-u_{0}\right) \\
& +\Phi\left(\eta, u_{0}\right)-\Phi\left(\eta, v_{i}\right)>0, \quad \forall i \in\{1, \ldots, n\}
\end{aligned}
$$


Let

$$
\begin{aligned}
\Lambda:=\{v \in D(\Phi(\eta, \cdot)): & \left\langle A u_{0}-f, u_{0}-v\right\rangle_{X} \\
& -J^{0}\left(\eta ; v-u_{0}\right) \\
& \left.+\Phi\left(\eta, u_{0}\right)-\Phi(\eta, v)>0\right\} .
\end{aligned}
$$

It is clear that $v_{i} \in \Lambda$ for all $i \in\{1, \ldots, n\}$. The convexity of the functions $\Phi(\eta, \cdot)$ and $J^{0}(\eta ; \cdot)$ implies that $\Lambda$ is a convex subset in $X$. We infer that $\operatorname{conv}\left\{v_{1}, \ldots, v_{n}\right\} \subset \Lambda$, so $u_{0} \in \Lambda$, which is obviously impossible. This contradiction justifies condition (ii) in Theorem 5. Thus all the assumptions of Theorem 5 are satisfied.

Applying Theorem 5, we obtain

$$
\bigcap_{v \in K \cap D(\Phi(\eta,))} G(v) \neq \emptyset .
$$

This ensures the existence of an element $u \in K \cap D(\Phi(\eta, \cdot))$ satisfying

$$
\begin{array}{r}
\langle A u, v-u\rangle_{X}+\Phi(\eta, v)-\Phi(\eta, u) \\
+J^{0}(\eta ; v-u) \geq\langle f, v-u\rangle_{X}
\end{array}
$$

for all $v \in K \cap D(\Phi(\eta, \cdot))$. The above inequality being also satisfied if $v \notin D(\Phi(\eta, \cdot))$, we conclude that $u$ is a solution of problem (13).

It remains to show that the solution of problem (13) is unique. If $u_{1}, u_{2} \in K$ are solutions of (13), then we have that $\left(\eta, u_{1}\right) \in D(\Phi),\left(\eta, u_{2}\right) \in D(\Phi)$, and

$$
\begin{aligned}
\left\langle A u_{1}, v-u_{1}\right\rangle_{X}+\Phi(\eta, v)-\Phi\left(\eta, u_{1}\right) & \\
+ & J^{0}\left(\eta ; v-u_{1}\right) \geq\left\langle f, v-u_{1}\right\rangle_{X}, \quad \forall v \in K, \\
\left\langle A u_{2}, v-u_{2}\right\rangle_{X}+\Phi(\eta, v)-\Phi\left(\eta, u_{2}\right) & \\
+ & J^{0}\left(\eta ; v-u_{2}\right) \geq\left\langle f, v-u_{2}\right\rangle_{X}, \quad \forall v \in K .
\end{aligned}
$$

Letting $v=u_{2}$ in the first inequality and $v=u_{1}$ in the second one and then adding the obtained relations, we arrive at

$$
\begin{aligned}
\left\langle A u_{1}\right. & \left.-A u_{2}, u_{2}-u_{1}\right\rangle_{X}+J^{0}\left(\eta ; u_{2}-u_{1}\right) \\
& +J^{0}\left(\eta ; u_{1}-u_{2}\right) \geq 0 .
\end{aligned}
$$

By assumption $\left(H_{3}\right)$, we conclude that $u_{1}=u_{2}$. The proof is complete.

Denote by $u_{\eta} \in K$ the unique solution of problem (13) corresponding to $\eta \in K$. Lemma 6 guarantees that $u_{\eta}$ exists and is unique. We define $\pi: K \rightarrow K$ by

$$
\pi(\eta)=u_{\eta}, \quad \forall \eta \in K .
$$

Lemma 7. Assume that hypotheses $\left(H_{1}\right)-\left(H_{3}\right)$ are fulfilled and that the closed, convex set $K$ is bounded in $X$. Then, the map $\pi: K \rightarrow K$ given in (23) is sequentially weakly continuous.
Proof. Let a sequence $\left\{\eta_{n}\right\}_{n \geq 1} \subset K$ such that $\eta_{n} \rightarrow \eta$ in $X$ for some $\eta \in K$. We need to show that $\pi\left(\eta_{n}\right) \rightarrow \pi(\eta)$ as $n \rightarrow \infty$. To do this, it suffices to check that, for any relabeled subsequence $\left\{\eta_{n}\right\}_{n \geq 1}$, there is a subsequence of $\left\{\pi\left(\eta_{n}\right)\right\}_{n \geq 1}$ weakly converging to $\pi(\eta)$.

By the compactness of the embedding of $X$ in $Y$, we have that $\eta_{n} \rightarrow \eta$ in $Y$. Denote, for simplicity, $\pi\left(\eta_{n}\right)=u_{n}$. The definition of $\pi$ yields $\left(\eta_{n}, u_{n}\right) \in D(\Phi)$ and

$$
\begin{aligned}
\left\langle A u_{n}, u_{n}-v\right\rangle_{X} & \\
\leq & \Phi\left(\eta_{n}, v\right)-\Phi\left(\eta_{n}, u_{n}\right)+J^{0}\left(\eta_{n} ; v-u_{n}\right) \\
& +\left\langle f, u_{n}-v\right\rangle_{X}, \quad \forall v \in K .
\end{aligned}
$$

Since $K$ is bounded, $\left\{u_{n}\right\}_{n \geq 1} \subset K$ and $X$ is reflexive, we know that along a subsequence, denoted again by $\left\{u_{n}\right\}_{n \geq 1}$, we have

$$
u_{n} \rightarrow w \text { in } X \text { as } n \longrightarrow \infty,
$$

for some $w \in X$. The first part of $\left(H_{2}\right)$ yields $(\eta, w) \in(K \times$ $K) \cap D(\Phi)$. Moreover, the compactness of the embedding of $X$ in $Y$ implies that $u_{n} \rightarrow w$ in $Y$. Letting $n \rightarrow \infty$ in (24), by means of $\left(H_{1}\right),\left(H_{2}\right)$, the convergences $\eta_{n} \rightarrow \eta$ and $u_{n} \rightarrow w$ in $Y$, and the upper semicontinuity of $J^{0}(\cdot ; \cdot)$ on $Y \times Y$, we get

$$
\begin{aligned}
\langle A w, w-v\rangle_{X} \leq & \limsup _{n \rightarrow \infty}\left\langle A u_{n}, u_{n}-v\right\rangle_{X} \\
\leq & \limsup _{n \rightarrow \infty}\left(\Phi\left(\eta_{n}, v\right)-\Phi\left(\eta_{n}, u_{n}\right)\right) \\
& +\limsup _{n \rightarrow \infty} J^{0}\left(\eta_{n} ; v-u_{n}\right)+\langle f, w-v\rangle_{X} \\
\leq & \Phi(\eta, v)-\Phi(\eta, w)+J^{0}(\eta ; v-w) \\
& +\langle f, w-v\rangle_{X}, \quad \forall v \in K .
\end{aligned}
$$

This means that $w \in K$ is a solution of problem (13). Lemma 6 ensures that $w$ is the unique solution of (13). Thus, by (23), we have $\pi(\eta)=w$. Taking into account (25), it follows that $\pi\left(\eta_{n}\right) \rightarrow \pi(\eta)$ as $n \rightarrow \infty$ up to a subsequence. This completes the proof.

Remark 8. As noted in the proof of Lemma 6, the closed, bounded, convex subset $K \subset X$ is metrizable for the weak topology. Therefore, Lemma 7 implies that $\pi$ is weakly continuous.

We need the following version of the Schauder fixed point theorem (see [10, page 452]).

Theorem 9. Suppose that

(i) $X$ is a reflexive, separable Banach space;

(ii) the map $T: M \subset X \rightarrow M$ is sequentially weakly continuous;

(iii) the set $M$ is nonempty, closed, bounded, and convex.

Then $\mathrm{T}$ has a fixed point. 
We are now in position to prove Theorem 2.

Proof of Theorem 2. In view of Lemma 7 and the assumptions on $X$ and $K$, we may apply Theorem 9 which shows that the map $\pi: K \rightarrow K$ admits a fixed point $u \in K$; that is, $\pi(u)=u$. Using the definition of $\pi$ (see (23)), we deduce that $u \in K$ is a solution of problem (1).

\section{Proof of Theorem 4}

It suffices to prove Theorem 4 when the set $K$ is unbounded because for a bounded set $K$ the result is true according to Theorem 2. Let $K_{m}=\left\{x \in K:\|x\|_{X} \leq m\right\}$. Let $m_{0} \geq 1$ be an integer such that $\left\|v_{0}\right\|_{X} \leq m_{0}$, where $v_{0}$ is the element entering $\left(H_{4}\right)$. We claim that Theorem 2 can be applied with $K$ replaced by $K_{m}$ whenever $m \geq m_{0}$.

Note that $v_{0} \in K_{m_{0}}$, so $v_{0} \in K_{m} \cap D(\Phi(\eta, \cdot))$ for all $\eta \in K$, all $m \geq m_{0}$ (using the first part of $\left(H_{4}\right)$ ). Thus, $K_{m} \cap D(\Phi(\eta, \cdot)) \neq \emptyset$ for all $\eta \in K_{m}$, all $m \geq m_{0}$. Since $K$ is convex and closed in $X$, it turns out that $K_{m}$ is convex, closed, and bounded in $X$, for all $m \geq m_{0}$.

We check that assumptions $\left(H_{1}\right)-\left(H_{3}\right)$ of Theorem 2 remain valid when $K$ is replaced by $K_{m}$ with $m \geq m_{0}$. Towards this, we fix some $m \geq m_{0}$. If $\left\{\left(\eta_{n}, u_{n}\right)\right\}_{n \geq 1} \subset\left(K_{m} \times K_{m}\right) \cap D(\Phi)$ satisfies $\eta_{n} \rightarrow \eta$ in $X$ and $u_{n} \rightarrow u$ in $X$, then assumption $\left(H_{2}\right)$ (for $K$ ) implies $(\eta, u) \in(K \times K) \cap D(\Phi)$. On the other hand, the weak convergences ensure that

$$
\|\eta\|_{X} \leq \liminf _{n \rightarrow \infty}\left\|\eta_{n}\right\|_{X} \leq m, \quad\|u\|_{X} \leq \liminf _{n \rightarrow \infty}\left\|u_{n}\right\|_{X} \leq m
$$

Hence, $(\eta, u) \in\left(K_{m} \times K_{m}\right) \cap D(\Phi)$. The second part of $\left(H_{2}\right)$ for $K_{m}$ and conditions $\left(H_{1}\right)$ and $\left(H_{3}\right)$ for $K_{m}$ hold because $\left(H_{1}\right)$, $\left(H_{2}\right)$, and $\left(H_{3}\right)$ have been imposed for $K$, which contains $K_{m}$. Thus it is permitted to apply Theorem 2 for $K_{m}$ in place of $K$, with any $m \geq m_{0}$.

Applying Theorem 2, we find a sequence $\left\{u_{m}\right\}_{m \geq m_{0}}$ in $X$ such that $u_{m} \in K_{m},\left(u_{m}, u_{m}\right) \in D(\Phi)$, and

$$
\begin{gathered}
\left\langle A u_{m}, v-u_{m}\right\rangle_{X}+\Phi\left(u_{m}, v\right)-\Phi\left(u_{m}, u_{m}\right) \\
+J^{0}\left(u_{m} ; v-u_{m}\right) \geq\left\langle f, v-u_{m}\right\rangle_{X}
\end{gathered}
$$

for all $v \in K_{m}$, all $m \geq m_{0}$. Letting $v=v_{0}$ (see $\left.\left(H_{4}\right)\right)$ in (28), we obtain

$$
\begin{aligned}
\left\langle A u_{m}, u_{m}-v_{0}\right\rangle_{X} \leq & \Phi\left(u_{m}, v_{0}\right)-\Phi\left(u_{m}, u_{m}\right) \\
& +J^{0}\left(u_{m} ; v_{0}-u_{m}\right)+\left\langle f, u_{m}-v_{0}\right\rangle_{X}
\end{aligned}
$$

for all $m \geq m_{0}$. By the definition of the convex subdifferential $\partial \Phi\left(u_{m}, \cdot\right)$, we have

$$
\begin{aligned}
& \Phi\left(u_{m}, v_{0}\right)-\Phi\left(u_{m}, u_{m}\right) \\
& \quad \leq\left\langle z, v_{0}-u_{m}\right\rangle_{X}, \quad \forall z \in \partial \Phi\left(u_{m}, \cdot\right)\left(v_{0}\right), \quad \forall m \geq m_{0}
\end{aligned}
$$

Then, invoking the growth condition for $\partial \Phi\left(u_{m}, \cdot\right)\left(v_{0}\right)$ in $\left(\mathrm{H}_{5}\right)$, we see that

$$
\Phi\left(u_{m}, v_{0}\right)-\Phi\left(u_{m}, u_{m}\right) \leq c_{0}\left(1+\left\|u_{m}\right\|_{X}^{p}\right), \quad \forall m \geq m_{0}
$$

Recall that

$$
J^{0}(u ; v)=\max _{w \in \partial J(u)}\langle w, v\rangle_{Y}, \quad \forall u, v \in Y
$$

(see [1, Proposition 2.1.2(b)]). This fact combined with the growth condition for the generalized gradient $\partial J\left(u_{m}\right)$ as stated in $\left(\mathrm{H}_{5}\right)$ enables us to write

$$
\begin{aligned}
J^{0}\left(u_{m} ; v_{0}-u_{m}\right) & =\max _{w \in \partial J\left(u_{m}\right)}\left\langle w, v_{0}-u_{m}\right\rangle_{Y} \\
& \leq c_{0}\left(1+\left\|u_{m}\right\|_{Y}^{p-1}\right)\left\|v_{0}-u_{m}\right\|_{Y}
\end{aligned}
$$

for all $m \geq m_{0}$. By the continuity of the embedding $X \subset Y$, the inequality above leads to

$$
\begin{aligned}
& J^{0}\left(u_{m} ; v_{0}-u_{m}\right) \\
& \quad \leq c_{1}\left(1+\left\|u_{m}\right\|_{X}^{p-1}\right)\left\|v_{0}-u_{m}\right\|_{X}, \quad \forall m \geq m_{0},
\end{aligned}
$$

where $c_{1}>0$ is a constant. Combining (29), (31), and (34) yields

$$
\begin{aligned}
& \left\langle A u_{m}, u_{m}-v_{0}\right\rangle_{X} \\
& \quad \leq c_{0}\left(1+\left\|u_{m}\right\|_{X}^{p}\right)+\left[c_{1}\left(1+\left\|u_{m}\right\|_{X}^{p-1}\right)+\|f\|_{X^{*}}\right]\left\|v_{0}-u_{m}\right\|_{X}
\end{aligned}
$$

for all $m \geq m_{0}$. Relation (35) ensures that the sequence $\left\{u_{m}\right\}_{m \geq m_{0}}$ is bounded in $X$; indeed, if we suppose that we have $\left\|u_{m}\right\|_{X} \rightarrow+\infty$ along a (relabeled) subsequence, then it is seen from (35) that there is a constant $c>0$ such that

$$
\limsup _{m \rightarrow \infty} \frac{\left\langle A u_{m}, u_{m}-v_{0}\right\rangle_{X}}{\left\|u_{m}\right\|_{X}^{p}} \leq c,
$$

which contradicts hypothesis $\left(H_{4}\right)$.

By the reflexivity of $X$, there exists a subsequence of $\left\{u_{m}\right\}_{m \geq m_{0}}$, denoted again by $\left\{u_{m}\right\}_{m \geq m_{0}}$, such that

$$
u_{m} \rightarrow u \text { in } X \text { as } m \longrightarrow \infty \text {, }
$$

for some $u \in X$. Using hypothesis $\left(H_{2}\right)$ with $\eta_{m}=u_{m}$, we derive that $(u, u) \in(K \times K) \cap D(\Phi)$.

It remains to show that $u$ verifies the inequality in problem (1). Let an arbitrary element $v \in K$ and let $m_{1}=$ $m_{1}(v) \in \mathbb{N}$ such that $m_{1} \geq \max \left\{m_{0},\|v\|_{X}\right\}$. Then $v \in K_{m}$ for each $m \geq m_{1}$ and so from (28), we have that

$$
\begin{aligned}
\left\langle A u_{m}, u_{m}-v\right\rangle_{X} \leq & \Phi\left(u_{m}, v\right)-\Phi\left(u_{m}, u_{m}\right) \\
& +J^{0}\left(u_{m} ; v-u_{m}\right)+\left\langle f, u_{m}-v\right\rangle_{X} .
\end{aligned}
$$


The compactness of the embedding $X \subset Y$ and (37) guarantee that $u_{m} \rightarrow u$ in $Y$ as $m \rightarrow \infty$. Then the upper semicontinuity of $J^{0}(\cdot ; \cdot)$ on $Y \times Y$ implies

$$
\limsup _{m \rightarrow \infty} J^{0}\left(u_{m} ; v-u_{m}\right) \leq J^{0}(u ; v-u) .
$$

Assumptions $\left(H_{1}\right)$ and $\left(H_{2}\right)$ ensure that

$$
\begin{gathered}
\langle A u, u-v\rangle_{X} \leq \limsup _{m \rightarrow \infty}\left\langle A u_{m}, u_{m}-v\right\rangle_{X}, \\
\limsup _{m \rightarrow \infty}\left(\Phi\left(u_{m}, v\right)-\Phi\left(u_{m}, u_{m}\right)\right) \leq \Phi(u, v)-\Phi(u, u) .
\end{gathered}
$$

Passing to the limsup as $m \rightarrow \infty$ in (38) and using (39) and (40), we get that $u \in K$ satisfies the inequality in (1). Since $v$ was chosen arbitrarily in $K$, we conclude that $u$ solves problem (1). The proof of Theorem 4 is complete.

\section{Acknowledgment}

This work is funded by a Marie Curie Intra-European Fellowship for Career Development within the European Community's 7th Framework Program (Grant Agreement no. PIEF-GA-2010-274519).

\section{References}

[1] F. H. Clarke, Optimization and Nonsmooth Analysis, vol. 5 of Classics in Applied Mathematics, SIAM, Philadelphia, Pa, USA, 2nd edition, 1990.

[2] P. Jebelean, D. Motreanu, and V. V. Motreanu, "A unified approach for a class of problems involving a pseudo-monotone operator," Mathematische Nachrichten, vol. 281, no. 9, pp. 12831293, 2008.

[3] B. Jadamba, A. A. Khan, and M. Sama, "Generalized solutions of quasi variational inequalities," Optimization Letters, vol. 6, no. 7, pp. 1221-1231, 2012.

[4] Z. Liu, "Generalized quasi-variational hemi-variational inequalities," Applied Mathematics Letters, vol. 17, no. 6, pp. 741-745, 2004.

[5] D. Motreanu and M. Sofonea, "Quasivariational inequalities and applications in frictional contact problems with normal compliance," Advances in Mathematical Sciences and Applications, vol. 10, no. 1, pp. 103-118, 2000.

[6] D. Goeleven and D. Motreanu, Variational and Hemivariational Inequalities: Theory, Methods and Applications. Vol. II. Unilateral Problems, vol. 70 of Nonconvex Optimization and its Applications, Kluwer Academic Publishers, Boston, Mass, USA, 2003.

[7] D. Motreanu and P. D. Panagiotopoulos, Minimax Theorems and Qualitative Properties of the Solutions of Hemivariational Inequalities, vol. 29 of Nonconvex Optimization and its Applications, Kluwer Academic Publishers, Dordrecht, The Netherlands, 1999.

[8] C. Baiocchi and A. Capelo, Variational and Quasivariational Inequalities. Applications to Free Boundary Problems, John Wiley \& Sons, New York, NY, USA, 1984.

[9] H. Brezis, Analyse Fonctionnelle. Théorie et Applications, Collection Mathématiques Appliquées pour la Maîtrise, Masson, Paris, 1983.
[10] E. Zeidler, Nonlinear Functional Analysis and Its Applications. Vol. I. Fixed-Point Theorems, Springer, New York, NY, USA, 1986. 


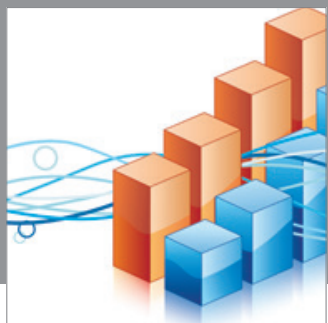

Advances in

Operations Research

mansans

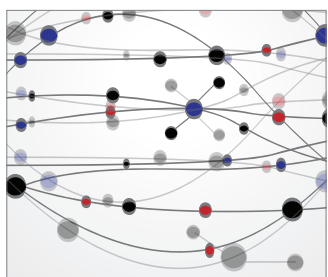

The Scientific World Journal
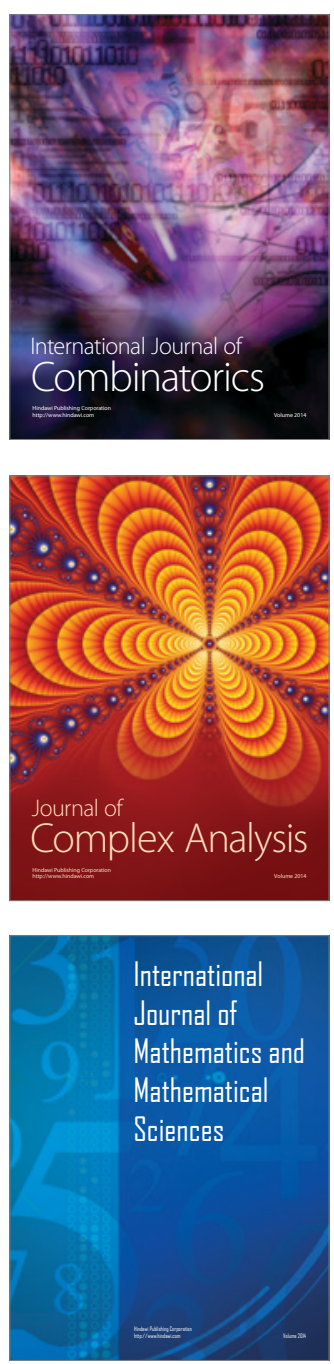
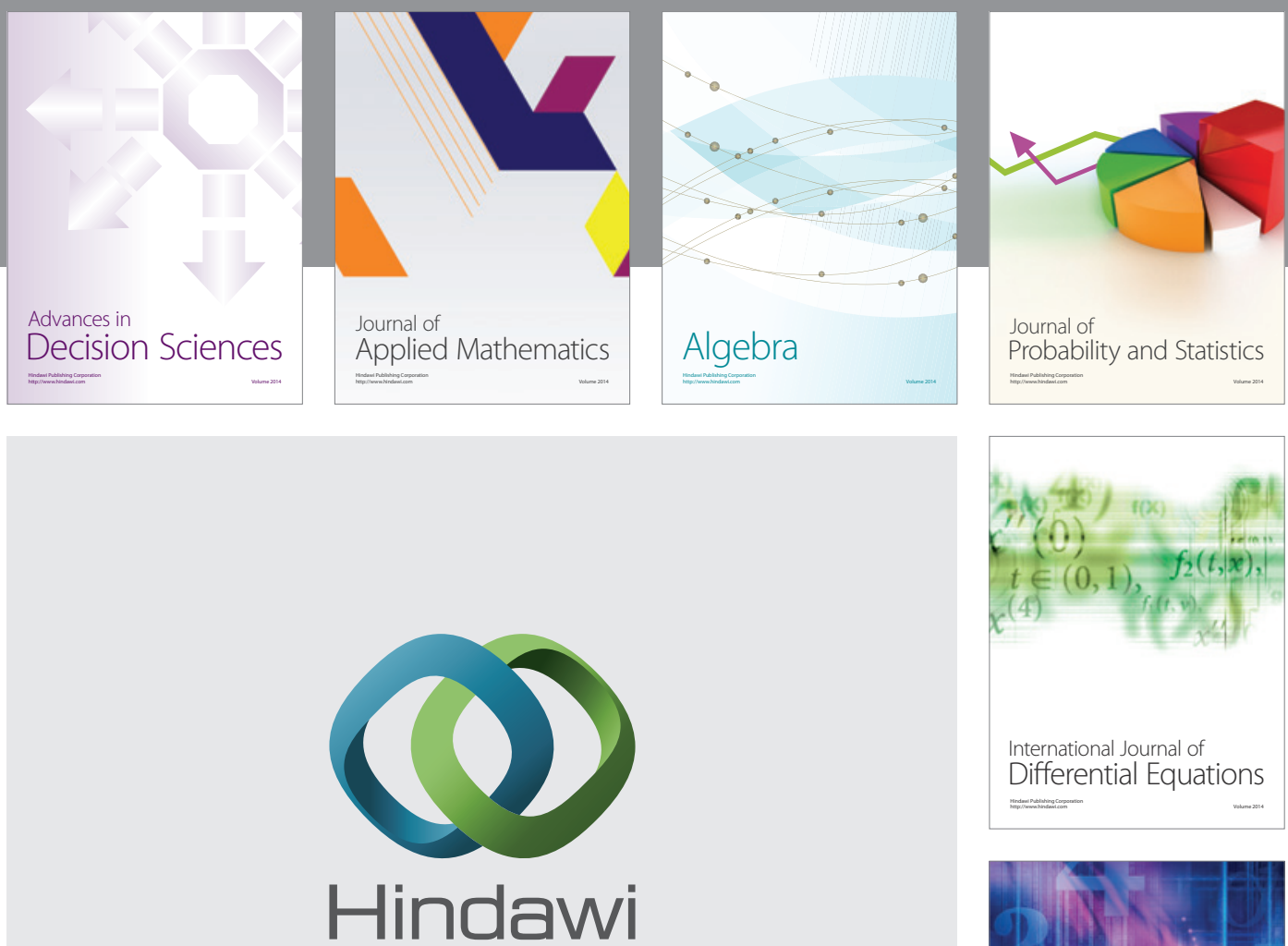

Submit your manuscripts at http://www.hindawi.com
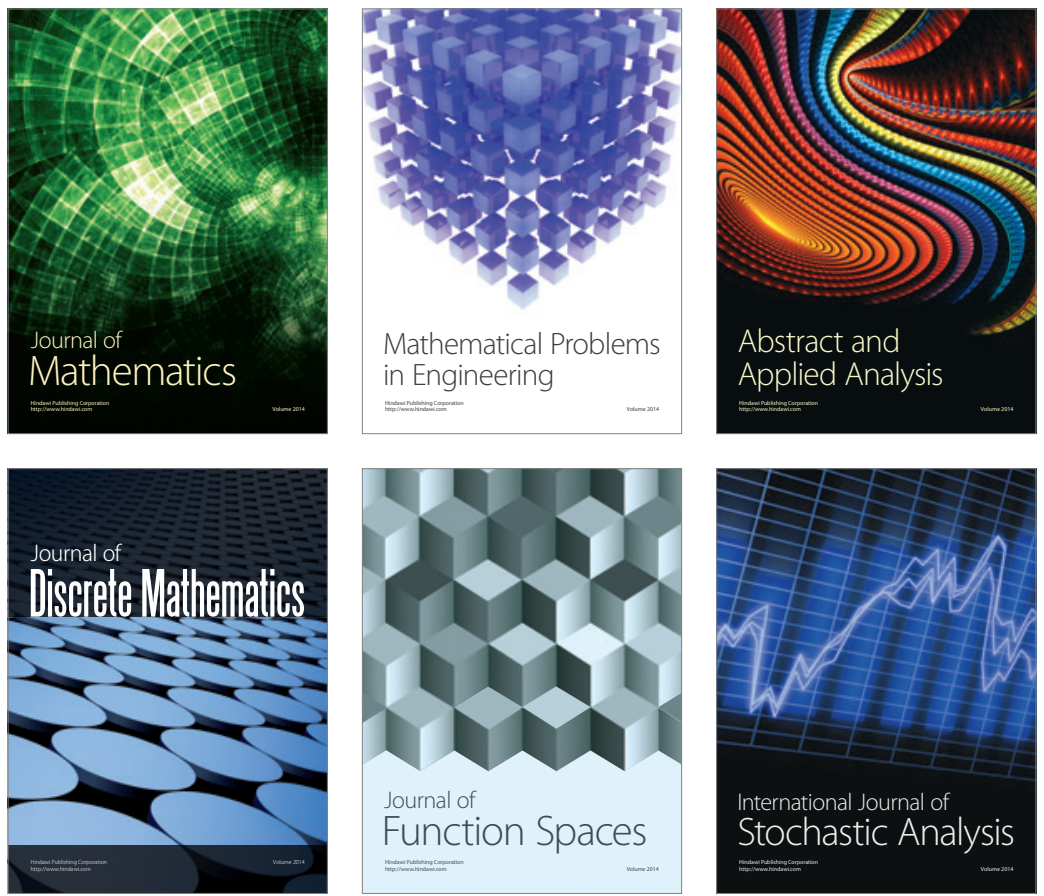

Journal of

Function Spaces

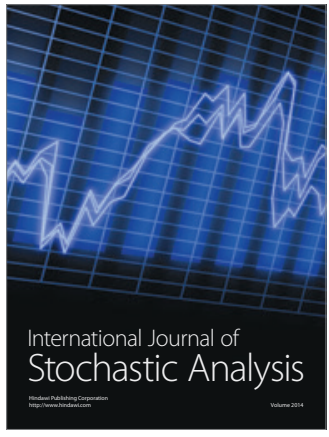

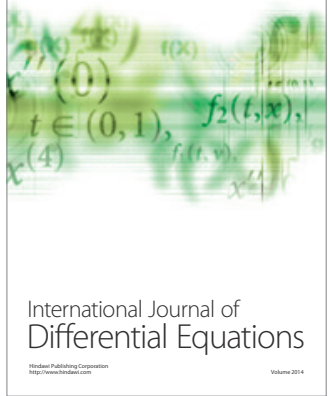
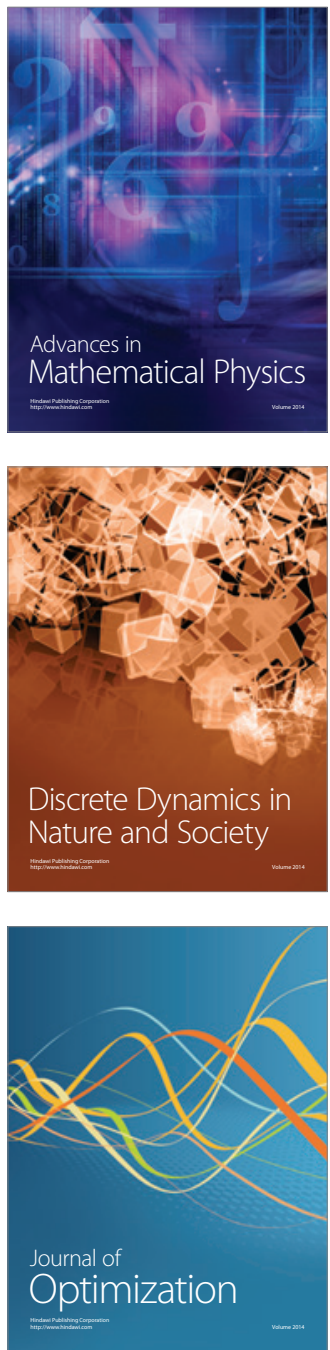